\title{
New periodic solutions of singular Hamiltonian systems with fixed energies
}

Fengying $\mathrm{Li}^{1 *}$, Qingqing $\mathrm{Hua}^{2}$ and Shiqing Zhang ${ }^{2}$

\section{*Correspondence:}

lify0308@163.com

'School of Economic and

Mathematics, Southwestern

University of Finance and

Economics, Chengdu, Sichuan

611130, P.R. China

Full list of author information is

available at the end of the article

\begin{abstract}
By using the variational minimizing method with a special constraint and the direct variational minimizing method without constraint, we study second-order Hamiltonian systems with a singular potential $V \in C^{2}\left(R^{n} \backslash O, R\right)$ and $V \in C^{1}\left(R^{2} \backslash O, R\right)$, which may have an unbounded potential well, and prove the existence of non-trivial periodic solutions with a prescribed energy. Our results can be regarded as complements of the well-known theorems of Benci-Gluck-Ziller-Hayashi and Ambrosetti-Coti Zelati and so on.
\end{abstract}

MSC: $35 \mathrm{~A} 15 ; 47 \mathrm{~J} 30$

Keywords: second-order singular Hamiltonian systems; periodic solutions; variational methods

\section{Introduction}

Seifert [1] in 1948 and Rabinowitz [2, 3] in 1978 and 1979 studied classical second-order Hamiltonian systems without singularity, based on their work, Benci $[4,5]$ and Gluck and Ziller [6] and Hayashi [7] used a Jacobi metric and very complicated geodesic methods and algebraic topology to study the periodic solutions with a fixed energy of the following system:

$$
\begin{aligned}
& \ddot{q}+V^{\prime}(q)=O, \\
& \frac{1}{2}|\dot{q}|^{2}+V(q)=h .
\end{aligned}
$$

They proved a very general theorem.

Theorem 1.1 Suppose $V \in C^{2}\left(R^{n}, R\right)$, if

$$
\left\{x \in R^{n} \mid V(x) \leq h\right\}
$$

is bounded and non-empty, then (1.1)-(1.2) has a periodic solution with energy $h$.

Furthermore, if

$$
V^{\prime}(x) \neq O, \quad \forall x \in\left\{x \in R^{n} \mid V(x)=h\right\},
$$

then (1.1)-(1.2) has a nonconstant periodic solution with energy $h$. 
For the existence of multiple periodic solutions for (1.1)-(1.2) with compact energy surfaces, we can refer to Groessen [8] and Long [9] and the references therein.

In the 1987 paper of Ambrosetti and Coti Zelati [10], Clark-Ekeland's dual action principle, Ambrosetti-Rabinowitz's mountain pass theorem etc. were used to study the existence of $T$-periodic solutions of the second-order equation

$$
-\ddot{x}=\nabla U(x),
$$

where

$$
U=V \in C^{2}(\Omega ; \mathbf{R})
$$

is such that

$$
U(x) \rightarrow \infty, \quad x \rightarrow \Gamma=\partial \Omega
$$

here $\Omega \subset \mathbf{R}^{n}$ is a bounded and convex domain, and they got the following result.

Theorem 1.2 Suppose that

(i) $U(O)=0=\min U$;

(ii) $U(x) \leq \theta(x, \nabla U(x))$ for some $\theta \in\left(0, \frac{1}{2}\right)$ and for all $x$ near $\Gamma$ (superquadraticity near $\Gamma)$;

(iii) $\left(U^{\prime \prime}(x) y, y\right) \geq k|y|^{2}$ for some $k>0$ and for all $(x, y) \in \Omega \times \mathbf{R}^{N}$.

Let $\omega_{N}$ be the greatest eigenvalue of $U^{\prime \prime}(0)$ and $T_{0}=\left(2 / \omega_{N}\right)^{1 / 2}$. Then $-\ddot{x}=\nabla U(x)$ has for each $T \in\left(0, T_{0}\right)$ a periodic solution with minimal period $T$.

For $C^{r}$ systems, a natural interesting problem is if

$$
\left\{x \in R^{n} \mid V(x) \leq h\right\}
$$

is unbounded: can we get a nonconstant periodic solution for the system (1.1)-(1.2)?

In 1987, Offin [11] firstly generalized Theorem 1.1 to some non-compact cases under $V \in C^{3}\left(R^{n}, R\right)$ and complicated geometrical assumptions on potential wells, but it seems to be difficult to verify this for concrete potentials under the geometrical conditions.

In 1988, Rabinowitz [12] studied multiple periodic solutions for classical Hamiltonian systems with potential $V \in C^{1}\left(R \times R^{n}, R\right)$, where $V\left(q_{1}, \ldots, q_{n} ; t\right)$ is $T_{i}$-periodic in positions $q_{i} \in R$ and is $T$-periodic in $t$.

In 1990, using Clark-Ekeland's dual variational principle and Ambrosetti-Rabinowitz's mountain pass lemma, Coti Zelati et al. [13] studied Hamiltonian systems with convex potential wells, they got the following result.

Theorem 1.3 Let $\Omega$ be a convex open subset of $R^{n}$ containing the origin $O$. Let $V \in C^{2}(\Omega, R)$ be such that

(V1) $V(q) \geq V(O)=0, \forall q \in \Omega$.

(V2) $\forall q \neq O, V^{\prime \prime}(q)>0$

(V3) $\exists \omega>0$, s.t. $V(q) \leq \frac{\omega}{2}\|q\|^{2}, \forall\|q\|<\epsilon$. 
(V4) $V^{\prime \prime}(q)^{-1} \rightarrow 0,\|q\| \rightarrow 0$, or

$(\mathrm{V} 4)^{\prime} V^{\prime \prime}(q)^{-1} \rightarrow 0, q \rightarrow \partial \Omega$.

Then, for every $T<\frac{2 \pi}{\sqrt{\omega}}$, (1.1) has a solution with minimal period $T$.

In Theorems 1.2 and 1.3, the authors assumed the convex conditions for potentials and potential wells so that they can apply Clark-Ekeland's dual variational principle; we notice that Theorems 1.1-1.3 essentially made the following assumption:

$$
V(x) \rightarrow \infty, \quad x \rightarrow \Gamma=\partial \Omega
$$

So all the potential wells are bounded.

For singular Hamiltonian systems with a fixed energy $h \in R$, Ambrosetti and Coti Zelati in $[14,15]$ used Ljusternik-Schnirelmann theory on a $C^{1}$ manifold to get the following theorem.

Theorem 1.4 (Ambrosetti and Coti Zelati [14]) Suppose $V \in C^{2}\left(R^{n} \backslash\{O\}, R\right)$ satisfies $V(q) \rightarrow-\infty, q \rightarrow 0$ and

$$
\begin{aligned}
& \text { (A1) } 3 V^{\prime}(u) \cdot u+\left(V^{\prime \prime}(u) u, u\right) \neq 0, \forall u \neq 0 \text {; } \\
& \text { (A2) } V^{\prime}(u) \cdot u>0, \forall u \neq 0 \text {; } \\
& \text { (A3) } \exists \alpha>2 \text {, s.t. } V^{\prime}(u) \cdot u \leq-\alpha V(u), \forall u \neq 0 \text {; } \\
& \text { (A4) } \exists \beta>2, r>0 \text {, s.t. } V^{\prime}(u) \cdot u \geq-\beta V(u), 0<|u|<r \text {; } \\
& \text { (A5) } V(u)+\frac{1}{2} V^{\prime}(u) u \leq 0, \forall u \neq 0 .
\end{aligned}
$$

Then (1.1)-(1.2) has at least one nonconstant periodic solution.

Besides Ambrosetti-Coti Zelati, many other mathematicians [16-34] studied singular Hamiltonian systems, here we only mention a related recent paper of Carminati, Sere and Tanaka [16]. They used complex variational and topological methods to generalize Pisani's results [17], and they got the following theorem.

Theorem 1.5 Suppose $h>0, L_{0}>0$ and $V \in C^{\infty}\left(R^{n} \backslash\{O\}, R\right)$ satisfies $V(q) \rightarrow-\infty, q \rightarrow 0$ and

(B1) $V(q) \leq 0, \forall q \neq 0$;

(B2) $V(q)+\frac{1}{2} V^{\prime}(q) q \leq h, \forall|q| \geq e^{L_{0}}$;

(B3) $V(q)+\frac{1}{2} V^{\prime}(q) q \geq h, \forall|q| \leq e^{-L_{0}}$;

(A4) $\exists \beta>2, r>0$, s.t. $V^{\prime}(q) \cdot q \geq-\beta V(q), 0<|q|<r$.

Then (1.1)-(1.2) has at least one periodic solution with the given energy $h$ and whose action is at most $2 \pi r_{0}$ with

$$
r_{0}=\max \left\{[2(h-V(q))]^{\frac{1}{2}} ;|q|=1\right\} .
$$

Theorem 1.6 Suppose $h>0, \rho_{0}>0$, and $V \in C^{\infty}\left(R^{n} \backslash\{O\}, R\right)$ satisfies $V(q) \rightarrow-\infty, q \rightarrow 0$ and (B1), (A4) and

(B2) $\lim _{|q| \rightarrow+\infty} V^{\prime}(q)=O$;

(B3)' $V(q)+\frac{1}{2} V^{\prime}(q) q \geq h, \forall|q| \leq \rho_{0}$.

Then (1.1)-(1.2) has at least one periodic solution with the given energy $h$ whose action is at most $2 \pi r_{0}$. 
By using the variational minimizing method with a special constraint, we obtain the following result.

Theorem 1.7 Suppose $V \in C^{2}\left(R^{n} \backslash\{O\}, R\right)$ and $V(q) \rightarrow-\infty, q \rightarrow 0$ and satisfies (A1)-(A3) and

(A4) $\exists \beta>2$, s.t. $V^{\prime}(q) \cdot q \geq-\beta V(q), 0<|q|<+\infty$;

$(\mathrm{A} 5)^{\prime} \quad V(-q)=V(q), \forall q \neq O$.

Then for any $h>0,(1.1)-(1.2)$ has at least one nonconstant periodic solution with the given energy $h$.

Using the direct variational minimizing method, we get the following theorem.

Theorem 1.8 Suppose $V \in C^{1}\left(R^{2} \backslash\{O\}, R\right)$ and $V(q) \rightarrow-\infty, q \rightarrow 0$ and satisfies

(B1) $V(q)<h, \forall q \neq O$;

$(\mathrm{P} 1)^{\prime} \quad V^{\prime}(u) \rightarrow O,\|u\| \rightarrow+\infty$;

(A3)' $\exists \alpha>2, \mu_{2}>0$, s.t. $V^{\prime}(u) \cdot u \leq-\alpha V(u)+\mu_{2}, \forall u \neq 0$;

(A4) $\exists \beta>2, r>0$, s.t. $V^{\prime}(u) \cdot u \geq-\beta V(u), 0<|u|<r$.

Then for any $h>\frac{\mu_{2}}{\alpha},(1.1)-(1.2)$ has at least one nonconstant periodic solution with the given energy $h$.

Corollary 1.9 Suppose $\alpha=\beta>2$ and

$$
V(x)=-|x|^{-\alpha} .
$$

Then for any $h>0$, (1.1)-(1.2) has at least one nonconstant periodic solution with the given energy $h$.

Remark In Theorem 1.8, the assumption on regularity for potential $V$ is weaker than Theorems 1.1-1.6. Comparing Theorem 1.5 with Theorem 1.8, our (B1)' is also weaker than (B1), and (A3)' is also different from (B2)-(B3) and (B3)'.

\section{A few lemmas}

Let

$$
H^{1}=W^{1,2}\left(R / Z, R^{n}\right)=\left\{u: R \rightarrow R^{n}, u \in L^{2}, \dot{u} \in L^{2}, u(t+1)=u(t)\right\} .
$$

Then the standard $H^{1}$ norm is equivalent to

$$
\|u\|=\|u\|_{H^{1}}=\left(\int_{0}^{1}|\dot{u}|^{2} d t\right)^{1 / 2}+|u(0)|
$$

Let

$$
\Lambda=\left\{u \in H^{1} \mid u(t) \neq O, \forall t\right\}
$$


Lemma 2.1 ([14]) Let

$$
F=\left\{u \in H^{1} \mid \int_{0}^{1}\left(V(u)+\frac{1}{2} V^{\prime}(u) u\right) d t=h\right\} .
$$

If (A1) holds, then $F$ is a $C^{1}$ manifold with codimension 1 in $H^{1}$. Let

$$
f(u)=\frac{1}{4} \int_{0}^{1}|\dot{u}|^{2} d t \int_{0}^{1} V^{\prime}(u) u d t
$$

and let $\tilde{u} \in F$ be such that $f^{\prime}(\widetilde{u})=O$ and $f(\widetilde{u})>0$. Set

$$
\frac{1}{T^{2}}=\frac{\int_{0}^{1} V^{\prime}(\tilde{u}) \tilde{u} d t}{\int_{0}^{1}|\dot{\widetilde{u}}|^{2} d t} .
$$

If (A2) holds, then $\widetilde{q}(t)=\widetilde{u}(t / T)$ is a nonconstant T-periodic solution for (1.1)-(1.2). Moreover, if (A2) holds, then $f(u) \geq 0$ on $F$ and $f(u)=0, u \in F$ if and only if $u$ is constant.

Lemma $2.2([8,14])$ Let $f(u)=\frac{1}{2} \int_{0}^{1}|\dot{u}|^{2} d t \int_{0}^{1}(h-V(u)) d t$ and $\widetilde{u} \in \Lambda$ be such that $f^{\prime}(\widetilde{u})=O$ and $f(\widetilde{u})>0$. Set

$$
\frac{1}{T^{2}}=\frac{\int_{0}^{1}(h-V(\widetilde{u})) d t}{\frac{1}{2} \int_{0}^{1}|\dot{\tilde{u}}|^{2} d t} .
$$

Then $\widetilde{q}(t)=\widetilde{u}(t / T)$ is a nonconstant T-periodic solution for (1.1)-(1.2). Furthermore, if $V(x)<h, \forall x \neq O$, then $f(u) \geq 0$ on $\Lambda$ and $f(u)=0, u \in \Lambda$ if and only if $u$ is a nonzero constant.

Lemma 2.3 (Sobolev-Rellich-Kondrachov [35, 36])

$$
W^{1,2}\left(R / Z, R^{n}\right) \subset C\left(R / Z, R^{n}\right)
$$

and the imbedding is compact.

Lemma 2.4 ([35, 36]) Let $q \in W^{1,2}\left(R / T Z, R^{n}\right)$.

(1) If $q(0)=q(T)=O$, then we have the Friedrics-Poincaré inequality:

$$
\int_{0}^{T}|\dot{q}(t)|^{2} d t \geq\left(\frac{\pi}{T}\right)^{2} \int_{0}^{T}|q(t)|^{2} d t
$$

(2) If $\int_{0}^{T} q(t) d t=0$, then we have Wirtinger's inequality:

$$
\int_{0}^{T}|\dot{q}(t)|^{2} d t \geq\left(\frac{2 \pi}{T}\right)^{2} \int_{0}^{T}|q(t)|^{2} d t
$$

and Sobolev's inequality:

$$
\int_{0}^{T}|\dot{q}(t)|^{2} d t \geq \frac{12}{T}|q(t)|_{\infty}^{2} .
$$


Lemma 2.5 (Eberlein-Shmulyan [37]) A Banach space $X$ is reflexive if and only if any bounded sequence in $X$ has a weakly convergent subsequence.

Definition 2.6 (Tonelli [35]) Let $X$ be a Banach space, $f: X \rightarrow R$.

(i) If for any $\left\{x_{n}\right\} \subset X$ strongly converges to $x_{0}: x_{n} \rightarrow x_{0}$, we have

$$
\liminf f\left(x_{n}\right) \geq f\left(x_{0}\right)
$$

then we call $f(x)$ lower semi-continuous at $x_{0}$.

(ii) If for any $\left\{x_{n}\right\} \subset X$ weakly converges to $x_{0}: x_{n} \rightarrow x_{0}$, we have

$$
\liminf f\left(x_{n}\right) \geq f\left(x_{0}\right)
$$

then we call $f(x)$ weakly lower semi-continuous at $x_{0}$.

Using the famous Ekeland variational principle, Ekeland proved the following.

Lemma 2.7 (Ekeland [38]) Let $X$ be a Banach space, $F \subset X$ be a closed (weakly closed) subset, let $\delta\left(x_{1}, x_{2}\right)$ be the geodesic distance between two points $x_{1}$ and $x_{2}$ in $X, \delta(x, F)$ be the geodesic distance between $x$ and the set $F$. Suppose that $\Phi$ defined on $X$ is Gateauxdifferentiable and lower semi-continuous (or weakly lower semi-continuous) and assume $\left.\Phi\right|_{F}$ restricted on $F$ is bounded from below. Then there is a sequence $\left\{x_{n}\right\} \subset F$ such that

$$
\begin{aligned}
& \delta\left(x_{n}, F\right) \rightarrow 0, \\
& \Phi\left(x_{n}\right) \rightarrow \inf _{F} \Phi, \\
& \left(1+\left\|x_{n}\right\|\right)\left\|\left.\Phi\right|_{F} ^{\prime}\left(x_{n}\right)\right\| \rightarrow 0 .
\end{aligned}
$$

Definition 2.8 ([38,39]) Let $X$ be a Banach space, $F \subset X$ be a closed subset. Suppose that $\Phi$ defined on $X$ is Gateaux-differentiable, if sequence $\left\{x_{n}\right\} \subset F$ is such that

$$
\begin{aligned}
& \delta\left(x_{n}, F\right) \rightarrow 0, \\
& \Phi\left(x_{n}\right) \rightarrow c, \\
& \left(1+\left\|x_{n}\right\|\right)\left\|\left.\Phi\right|_{F} ^{\prime}\left(x_{n}\right)\right\| \rightarrow 0,
\end{aligned}
$$

then $\left\{x_{n}\right\}$ has a strongly convergent subsequence.

Then we say that $f$ satisfies the $(C P S)_{c, F}$ condition at the level $c$ for the closed subset $F \subset X$.

We notice that if $F=X$, then the above condition is the classical Cerami-Palais-Smale condition [40].

We can give a weaker condition than the $(C P S)_{c, F}$ condition.

Definition 2.9 Let $X$ be a Banach space, $F \subset X$ be a weakly closed subset. Suppose that $\Phi$ defined on $X$ is Gateaux-differentiable, if sequence $\left\{x_{n}\right\} \subset F$ such that

$$
\delta\left(x_{n}, F\right) \rightarrow 0
$$




$$
\begin{aligned}
& \Phi\left(x_{n}\right) \rightarrow c, \\
& \left\|\left.\Phi\right|_{F} ^{\prime}\left(x_{n}\right)\right\| \rightarrow 0,
\end{aligned}
$$

then $\left\{x_{n}\right\}$ has a weakly convergent subsequence.

Then we say that $f$ satisfies the $(W C P S)_{c, F}$ condition.

Lemma 2.10 (Gordon [18]) Let $V$ satisfy the so-called Gordon strong force condition: There exists a neighborhood $\mathcal{N}$ of $O$ and a function $U \in C^{1}(\Omega, \mathbb{R})$ such that:

(i) $\lim _{s \rightarrow 0} U(x)=-\infty$;

(ii) $-V(x) \geq\left|U^{\prime}(x)\right|^{2}$ for every $x \in \mathcal{N}-\{O\}$.

Let

$$
\partial \Lambda=\left\{u \in H^{1}=W^{1,2}\left(R / Z, R^{n}\right), \exists t_{0}, u\left(t_{0}\right)=O\right\} .
$$

Then we have

$$
\int_{0}^{1} V(u) d t \rightarrow-\infty, \quad \forall u_{n} \rightarrow u \in \partial \Lambda .
$$

Let

$$
\partial \Lambda=\left\{u \in H^{1}=W^{1,2}\left(R / Z, R^{n}\right), \exists t_{0}, u\left(t_{0}\right)=0\right\} .
$$

Then we have

$$
\int_{0}^{1} V(u) d t \rightarrow-\infty, \quad \forall u_{n} \rightarrow u \in \partial \Lambda .
$$

By Lemmas 2.7 and 2.10 , it is easy to prove the following.

Lemma 2.11 Let $X$ be a Banach space, let $F \subset X$ be a weakly closed subset. Suppose that $\Phi$ defined on $F$ is Gateaux-differentiable and weakly lower semi-continuous and bounded from below on $F$. If $\Phi$ satisfies the $(C P S)_{\mathrm{in} \Phi, F}$ condition or the $(W C P S)_{\mathrm{inf} \Phi, F}$ condition, and suppose that

$$
\Phi\left(u_{n}\right) \rightarrow+\infty, \quad u_{n} \rightarrow u \in \partial \Lambda,
$$

then $\Phi$ attains its infimum on $F$.

The next lemma is a variant on the classical Tonelli's theorem, whose proof is easy, so we omit its proof.

Lemma 2.12 Let $X$ be a Banach space, $F \subset X$ be a weakly closed subset. Suppose that $\phi(u)$ is defined on an open subset $\Lambda \subset X$ and is Gateaux-differentiable on $\Lambda$ and weakly lower semi-continuous and bounded from below on $\Lambda \cap F$, if $\phi$ is coercive, that is, $\phi(x) \rightarrow+\infty$ as $\|x\| \rightarrow+\infty$, and suppose that

$$
\phi\left(u_{n}\right) \rightarrow+\infty, \quad u_{n} \rightarrow u \in \partial \Lambda,
$$

then $\phi$ attains its infimum on $\Lambda \cap F$. 


\section{The proof of Theorem 1.7}

By the symmetrical condition (A5)', it is easy to prove that the critical point of the functional $f$ on $\Lambda_{0}$ is also the critical point of the functional $f$ on $\Lambda$.

Let

$$
\partial \Lambda_{0}=\left\{u \in H^{1}=W^{1,2}\left(R / Z, R^{n}\right), u(t+1 / 2)=-u(t), \exists t_{0}, u\left(t_{0}\right)=0\right\} .
$$

Lemma 3.1 Assume (A4)' holds, then for any weakly convergent sequence $u_{n} \rightarrow u \in \partial \Lambda_{0}$, we have

$$
f\left(u_{n}\right) \rightarrow+\infty
$$

Proof Similar to the proof of Zhang [19].

Lemma 3.2 $F \cap \Lambda$ is a weakly closed subset in $H^{1}$.

Proof Let $\left\{u_{n}\right\} \subset F \cap \Lambda$ be a weakly convergent sequence, we use the embedding theorem to find which uniformly converges to $u \in H^{1}$.

Now we claim $u \in \Lambda$, and then it is obvious that $u \in F$. In fact, if $u \in \partial \Lambda$, by $V(q) \rightarrow-\infty$, $q \rightarrow 0$ and the condition (A4)' we have

$$
-V(u) \geq C_{1}|u|^{-\beta}, \quad 0<|u|<r^{\prime}<r .
$$

So $V(u)$ satisfies Gordon's strong force condition, and by his lemma, we have

$$
\int_{0}^{1}-V\left(u_{n}\right) d t \rightarrow+\infty, \quad \forall u_{n} \rightarrow u \in \partial \Lambda
$$

The condition (A4)' implies

$$
V\left(u_{n}\right)+\frac{1}{2}\left\langle V^{\prime}\left(u_{n}\right), u_{n}\right\rangle \geq\left(1-\frac{\beta}{2}\right) V\left(u_{n}\right) .
$$

Hence

$$
h=\int_{0}^{1}\left[V\left(u_{n}\right)+\frac{1}{2}\left\langle V^{\prime}\left(u_{n}\right), u_{n}\right\rangle\right] d t \rightarrow+\infty .
$$

This is a contradiction.

Lemma 3.3 $f(u)$ is weakly lower semi-continuous on $F \cap \Lambda_{0}$

Proof For any $\left\{u_{n}\right\} \subset F: u_{n} \rightarrow u$, then by Sobolev's embedding theorem and functional analysis, we have uniform convergence:

$$
\left|u_{n}(t)-u(t)\right|_{\infty} \rightarrow 0
$$


(i) If $u \in \Lambda_{0}$, then by $V \in C^{1}\left(R^{n} \backslash\{0\}, R\right)$, we have

$$
\mid V\left(u_{n}(t)\right)-V\left(\left.u(t)\right|_{\infty} \rightarrow 0\right.
$$

It's well known that the norm is weakly lower semi-continuous, we have

$$
\liminf \left\|u_{n}\right\| \geq\|u\| .
$$

Hence

$$
\begin{aligned}
\liminf f\left(u_{n}\right) & =\liminf \left(\frac{1}{2} \int_{0}^{1}\left|\dot{u}_{n}\right|^{2} d t\right) \int_{0}^{1}\left(h-V\left(u_{n}\right)\right) d t, \\
& \geq \frac{1}{2} \int_{0}^{1}|\dot{u}|^{2} d t \int_{0}^{1}(h-V(u)) d t=f(u) .
\end{aligned}
$$

(ii) If $u \in \partial \Lambda_{0}$, then by our assumption on $V$ which satisfies Gordon's strong force condition, we have

$$
\int_{0}^{1}-V\left(u_{n}\right) d t \rightarrow+\infty, \quad \forall u_{n} \rightarrow u \in \partial \Lambda_{0} .
$$

(1) If $u \equiv 0$, then

$$
\left|u_{n}\right|_{\infty} \rightarrow 0, \quad n \rightarrow+\infty
$$

Then similar to the proof in [19], we have

$$
f\left(u_{n}\right) \geq 6\left|u_{n}\right|_{\infty}^{2-\beta} \rightarrow+\infty, \quad n \rightarrow+\infty .
$$

So in this case we have

$$
\liminf f\left(u_{n}\right)=+\infty \geq f(u) .
$$

(2) If $u \neq 0$, then by the weakly lower semi-continuity for norm, we have

$$
\liminf \left\|u_{n}\right\| \geq\|u\|>0 .
$$

So by Gordon's lemma, we have

$$
\begin{aligned}
\liminf f\left(u_{n}\right) & =\liminf \left(\frac{1}{2} \int_{0}^{1}\left|\dot{u}_{n}\right|^{2} d t\right) \int_{0}^{1}\left(h-V\left(u_{n}\right)\right) d t=+\infty \\
& \geq \frac{1}{2} \int_{0}^{1}|\dot{u}|^{2} d t \int_{0}^{1}(h-V(u)) d t=f(u) .
\end{aligned}
$$

Lemma 3.4 The functional $f(u)$ has a positive lower bound on $F$.

Proof By the definitions of $f(u)$ and $F$ and the assumption (A2), we have

$$
f(u)=\frac{1}{4} \int_{0}^{1}|\dot{u}|^{2} d t \int_{0}^{1}\left(V^{\prime}(u) u\right) d t \geq 0, \quad \forall u \in F .
$$


By the definitions of the functional $f(u)$ and its domain $\Lambda_{0}$, and the conditions on the energy $h>0$ and the potential $V(u)<0$, it is easy to prove the following lemma.

Lemma 3.5 The functional $f(u)$ is coercive.

Furthermore, we claim that

$$
c=\inf _{F \cap \Lambda_{0}} f(u)>0,
$$

since otherwise, $u_{0}(t)=$ const attains the infimum 0 , then by the symmetry of $\Lambda_{0}$, we have $u_{0}(t) \equiv o$, which contradicts the definition of $\Lambda_{0}$. Now by Lemmas 3.1-3.4 and Lemmas 2.11 and 2.12 , we know $f(u)$ attains the infimum on $F$, furthermore we know that the minimizer is nonconstant.

\section{The proof of Theorem 1.8}

In order to prove the Cerami-Palais-Smale type condition and get a nonconstant periodic solution in non-symmetrical case, we need to add a topological condition, we know that there are winding numbers (degrees) in the planar case, so we define

$$
\Lambda_{1}=\{u \in \Lambda, \operatorname{deg}(u) \neq 0\} .
$$

Lemma 4.1 If $u_{n} \rightarrow u \in \partial \Lambda_{1}$, then $f\left(u_{n}\right) \rightarrow+\infty$.

Proof By $V$ satisfying Gordon's strong force condition, we have

$$
\int_{0}^{1}-V\left(u_{n}\right) d t \rightarrow+\infty, \quad \forall u_{n} \rightarrow u \in \partial \Lambda_{1}
$$

(1) If $u \equiv 0$, then by Sobolev's embedding theorem, we have

$$
\left|u_{n}\right|_{\infty} \rightarrow 0, \quad n \rightarrow+\infty
$$

Then by $\operatorname{deg}\left(u_{n}\right) \neq 0$, we have $c>0$ such that

$$
c\left|u_{n}\right|_{\infty} \leq\left\|\dot{u}_{n}\right\|_{L^{2}}
$$

and $\left\|\dot{u}_{n}\right\|_{L^{2}}$ is an equivalent norm of $W^{1,2}$ and

$$
f\left(u_{n}\right) \geq c\left|u_{n}\right|_{\infty}^{2-\beta} \rightarrow+\infty, \quad n \rightarrow+\infty
$$

So in this case, we have

$$
\liminf f\left(u_{n}\right)=+\infty \geq f(u)
$$

(2) If $u \neq 0$, then by the weakly lower semi-continuity for the norm, we have

$$
\liminf \left\|u_{n}\right\| \geq\|u\|>0
$$


So by Gordon's lemma, we have

$$
\begin{aligned}
\liminf f\left(u_{n}\right) & =\liminf \left(\frac{1}{2} \int_{0}^{1}\left|\dot{u}_{n}\right|^{2} d t\right) \int_{0}^{1}\left(h-V\left(u_{n}\right)\right) d t=+\infty \\
& =\frac{1}{2} \int_{0}^{1}|\dot{u}|^{2} d t \int_{0}^{1}(h-V(u)) d t=f(u) .
\end{aligned}
$$

Lemma 4.2 Under the assumptions of Theorem 1.8,

$$
f(u)=\frac{1}{2} \int_{0}^{1}|\dot{u}|^{2} d t \int_{0}^{1}(h-V(u)) d t
$$

satisfies the $(C P S)^{+}$condition on $\Lambda_{1}$, that is, if $\left\{u_{n}\right\} \subset \Lambda_{1}$ satisfies

$$
f\left(u_{n}\right) \rightarrow c>0, \quad\left(1+\left\|u_{n}\right\|\right) f^{\prime}\left(u_{n}\right) \rightarrow O,
$$

then $\left\{u_{n}\right\}$ has a strongly convergent subsequence in $\Lambda_{1}$.

Proof Since $f^{\prime}\left(u_{n}\right)$ makes sense, we know

$$
\left\{u_{n}\right\} \subset \Lambda_{1} .
$$

We claim $\int_{0}^{1}\left|\dot{u}_{n}\right|^{2} d t$ is bounded. In fact, by $f\left(u_{n}\right) \rightarrow c$, we have

$$
-\frac{1}{2}\left\|\dot{u}_{n}\right\|_{L^{2}}^{2} \cdot \int_{0}^{1} V\left(u_{n}\right) d t \rightarrow c-\frac{h}{2}\left\|\dot{u}_{n}\right\|_{L^{2}}^{2} .
$$

By $(\mathrm{A} 3)^{\prime}$ we have

$$
\begin{aligned}
\left\langle f^{\prime}\left(u_{n}\right), u_{n}\right\rangle & =\left\|\dot{u}_{n}\right\|_{L^{2}}^{2} \cdot \int_{0}^{1}\left(h-V\left(u_{n}\right)-\frac{1}{2}\left\langle V^{\prime}\left(u_{n}\right), u_{n}\right\rangle\right) d t \\
& \geq\left\|\dot{u}_{n}\right\|_{L^{2}}^{2} \int_{0}^{1}\left[h-\frac{\mu_{2}}{2}-\left(1-\frac{\alpha}{2}\right) V\left(u_{n}\right)\right] d t .
\end{aligned}
$$

By (4.2) and (4.3) we have

$$
\begin{aligned}
\left\langle f^{\prime}\left(u_{n}\right), u_{n}\right\rangle & \geq\left(h-\frac{\mu_{2}}{2}\right)\left\|\dot{u}_{n}\right\|_{L^{2}}^{2}+\left(1-\frac{\alpha}{2}\right)\left(2 c-h\left\|\dot{u}_{n}\right\|_{L^{2}}^{2}\right) \\
& =\left(\frac{\alpha}{2} h-\frac{\mu_{2}}{2}\right)\left\|\dot{u}_{n}\right\|_{L^{2}}^{2}+C_{1},
\end{aligned}
$$

where $C_{1}=2\left(1-\frac{\alpha}{2}\right) c, \alpha>2, h>\frac{\mu_{2}}{\alpha}$. So $\left\|\dot{u}_{n}\right\|_{2} \leq C_{2}$.

Then we claim $\left|u_{n}(0)\right|$ is bounded.

We notice that

$$
\begin{aligned}
& f^{\prime}\left(u_{n}\right) \cdot\left(u_{n}-u_{n}(0)\right) \\
& \quad=\int_{0}^{1}\left|\dot{u}_{n}\right|^{2} d t \int_{0}^{1}\left(h-V\left(u_{n}\right)\right) d t
\end{aligned}
$$




$$
\begin{aligned}
& -\frac{1}{2} \int_{0}^{1}\left|\dot{u}_{n}\right|^{2} d t \int_{0}^{1}\left\langle V^{\prime}\left(u_{n}\right), u_{n}-u_{n}(0)\right\rangle d t \\
= & 2 f\left(u_{n}\right)-\frac{1}{2} \int_{0}^{1}\left|\dot{u}_{n}\right|^{2} \int_{0}^{1}\left\langle V^{\prime}\left(u_{n}\right), u_{n}-u_{n}(0)\right\rangle d t .
\end{aligned}
$$

If $\left|u_{n}(0)\right|$ is unbounded, then there is a subsequence, still denoted by $u_{n}$ s.t. $\left|u_{n}(0)\right| \rightarrow+\infty$. Since

$$
\left\|\dot{u}_{n}\right\| \leq M_{1}
$$

we have

$$
\min _{0 \leq t \leq 1}\left|u_{n}(t)\right| \geq\left|u_{n}(0)\right|-\left\|\dot{u}_{n}\right\|_{2} \rightarrow+\infty, \quad \text { as } n \rightarrow+\infty
$$

By Friedrics-Poincaré's inequality and the condition (P1), we have

$$
\begin{aligned}
& \int_{0}^{1}\left|\dot{u}_{n}(t)\right|^{2} d t \geq \pi^{2} \int_{0}^{1}\left|u_{n}(t)-u_{n}(0)\right|^{2} d t \\
& \int_{0}^{1} V^{\prime}\left(u_{n}\right)\left(u_{n}-u_{n}(0)\right) d t \rightarrow 0 \\
& f^{\prime}\left(u_{n}\right) \cdot\left(u_{n}-u_{n}(0)\right) \rightarrow 0
\end{aligned}
$$

So $f\left(u_{n}\right) \rightarrow 0$, which contradicts $f\left(u_{n}\right) \rightarrow c>0$, hence $u_{n}(0)$ is bounded, and $\left\|u_{n}\right\|=$ $\left\|\dot{u}_{n}\right\|_{L^{2}}+\left|u_{n}(0)\right|$ is bounded. Furthermore, similar to the proof of Ambrosetti and Coti Zelati [15], $u_{n}$ strongly converges to $u \in \Lambda$.

It is easy to prove the following.

Lemma 4.3 Under the assumption $(\mathrm{B} 1)^{\prime}, f(u) \geq 0$ on $\Lambda$, that is, $f$ has a lower bound.

Lemma 4.4 Under the assumptions of Theorem $1.8, f(u)$ is weakly lower semi-continuous on the closure $\bar{\Lambda}$ of $\Lambda$.

Now we can prove our Theorem 1.8, in fact, by Lemma 4.1, we know that the infimum of $f$ on $\Lambda_{1}$ is equal to the infimum of $f$ on the closure of $\Lambda_{1}$. Furthermore, we can prove the infimum of $f$ on $\Lambda_{1}$ is greater than zero, otherwise if it is zero, the corresponding minimizer must be constant, then the winding number is zero, which is a contradiction. Now by the above lemmas, especially Lemma 2.11, we know that $f$ attains the positive infimum on $\Lambda_{1}$ and the corresponding minimizer must be nonconstant.

\section{Competing interests}

The authors declare that they have no competing interests.

Authors' contributions

The research and writing of this manuscript was a collaborative effort made by all the authors. All authors read and approved the final manuscript.

\section{Author details}

${ }^{1}$ School of Economic and Mathematics, Southwestern University of Finance and Economics, Chengdu, Sichuan 611130

P.R. China. ${ }^{2}$ Department of Mathematics, Sichuan University, Chengdu, Sichuan 610068, P.R. China. 


\section{Acknowledgements}

The authors would like to thank the editor and the referees for their many valuable comments. This paper was partially supported by NSF of China and the Grant for the Advisors of PhD students.

\section{Received: 1 July 2014 Accepted: 16 September 2014 Published: 16 Oct 2014}

\section{References}

1. Seifert, H: Periodische bewegungen mechanischer systeme. Math. Z. 51, 197-216 (1948)

2. Rabinowitz, PH: Periodic solutions of Hamiltonian systems. Commun. Pure Appl. Math. 31, 157-184 (1978)

3. Rabinowitz, PH: Periodic solutions of a Hamiltonian systems on a prescribed energy surface. J. Differ. Equ. 33, 336-352 (1979)

4. Benci, V: Normal modes of a Lagrangian system constrained in a potential well. Ann. Inst. Henri Poincaré, Anal. Non Linéaire 1, 379-400 (1984)

5. Benci, $\mathrm{V}$ : Closed geodesics for the Jacobi metric and periodic solutions of prescribed energy of natural Hamiltonian systems. Ann. Inst. Henri Poincaré, Anal. Non Linéaire 1, 401-412 (1984)

6. Gluck, H, Ziller, W: Existence of periodic motions of conservative systems. In: Bombieri, E (ed.) Seminar on Minimal Submanifolds. Princeton University Press, Princeton (1983)

7. Hayashi, K: Periodic solutions of classical Hamiltonian systems. Tokyo J. Math. 6, 473-486 (1983)

8. Van Groesen, EWC: Analytical mini-max methods for Hamiltonian break orbits with a prescribed energy. J. Math. Anal. Appl. 132, 1-12 (1988)

9. Long, Y: Index Theory for Symplectic Paths with Applications. Birkhäuser, Basel (2002)

10. Ambrosetti, A, Coti Zelati, V: Solutions with minimal period for Hamiltonian systems in a potential well. Ann. Inst. Henri Poincaré, Anal. Non Linéaire 4, 275-296 (1987)

11. Offin, D: A class of periodic orbits in classical mechanics. J. Differ. Equ. 66, 90-117 (1987)

12. Rabinowitz, PH: On a class of functionals invariant under a $Z^{n}$ action. Trans. Am. Math. Soc. 310, 303-311 (1988)

13. Coti Zelati, V, Ekeland, I, Lions, PL: Index estimates and critical points of functionals not satisfying Palais-Smale. Ann. Sc. Norm. Super. Pisa 17, 569-581 (1990)

14. Ambrosetti, A, Coti Zelati, V: Closed orbits of fixed energy for singular Hamiltonian systems. Arch. Ration. Mech. Anal. 112, 339-362 (1990)

15. Ambrosetti, A, Coti Zelati, V: Periodic Solutions for Singular Lagrangian Systems. Springer, Berlin (1993)

16. Carminati, C, Sere, E, Tanaka, K: The fixed energy problem for a class of nonconvex singular Hamiltonian systems. J. Differ. Equ. 230, 362-377 (2006)

17. Pisani, L: Periodic solutions with prescribed energy for singular conservative systems involving strong forces. Nonlinear Anal. TMA 21, 167-179 (1993)

18. Gordon, WB: Conservative dynamical systems involving strong forces. Trans. Am. Math. Soc. 204, 113-135 (1975)

19. Zhang, SQ: Multiple geometrically distinct closed noncollision orbits of fixed energy for $N$-body type problems with strong force potentials. Proc. Am. Math. Soc. 124, 3039-3046 (1996)

20. Benci, V, Giannoni, G: Periodic solutions of prescribed energy for a class of Hamiltonian system with singular potentials. J. Differ. Equ. 82, 60-70 (1989)

21. Chang, KC: Infinite Dimensional Morse Theory and Multiple Solution Problems. Birkhäuser, Basel (1993)

22. Degiovanni, M, Giannoni, F: Dynamical systems with Newtonian type potentials. Ann. Sc. Norm. Super. Pisa 15, 467-494 (1988)

23. Fadell, E, Husseini, S: A note on the category of free loop space. Proc. Am. Math. Soc. 102, 527-536 (1989)

24. Ambrosetti, A, Coti Zelati, V: Critical points with lack of compactness and applications to singular dynamical system. Ann. Mat. Pura Appl. 149, 237-259 (1987)

25. Greco, C: Periodic solutions of a class of singular Hamiltonian systems. Nonlinear Anal. TMA 12, 259-269 (1988)

26. Majer, P: Ljusternik-Schnirelmann theory with local Palais-Smale conditions and singular dynamical systems. Ann. Inst. Henri Poincaré, Anal. Non Linéaire 8, 459-476 (1991)

27. Rabinowitz, PH: A note on periodic solutions of prescribed energy for singular Hamiltonian systems. J. Comput. Appl. Math. 52, 147-154 (1994)

28. Serra, E, Terracini, S: Noncollision solutions to some singular minimization problems with Keplerian-like potentials. Nonlinear Anal. TMA 22, 45-62 (1994)

29. Tanaka, K: A prescribed energy problem for a singular Hamiltonian system with weak force. J. Funct. Anal. 113, 351-390 (1993)

30. Tanaka, K: A prescribed energy problem for conservative singular Hamiltonian system. Arch. Ration. Mech. Anal. 128, 127-164 (1994)

31. Tanaka, K: Periodic solutions for singular Hamiltonian systems and closed geodesics on non-compact Riemannian manifolds. Ann. Inst. Henri Poincaré, Anal. Non Linéaire 17, 1-33 (2000)

32. Terracini, S: Multiplicity of periodic solutions of prescribed energy problem for singular dynamical system. Ann. Inst. Henri Poincaré, Anal. Non Linéaire 9, 597-641 (1992)

33. Ambrosetti, A, Struwe, M: Periodic motions of conservative systems with singular potentials. NoDEA Nonlinear Differ. Equ. Appl. 1, 179-202 (1998)

34. Bahri, A, Rabinowitz, PH: A minimax method for a class of Hamiltonian systems with singular potentials. J. Funct. Anal. $82,412-428(1989)$

35. Mawhin, J, Willem, M: Critical Point Theory and Applications. Springer, Berlin (1989)

36. Ziemer, WP: Weakly Differentiable Functions. Springer, Berlin (1989)

37. Yosida, K: Functional Analysis. Springer, Berlin (1978)

38. Ekeland, I: Convexity Methods in Hamiltonian Mechanics. Springer, Berlin (1990)

39. Ghoussoub, N, Preiss, D: A general mountain pass principle for locating and classifying critical points. Ann. Inst. Henri Poincaré, Anal. Non Linéaire 6, 321-330 (1989)

40. Cerami, G: Un criterio di esistenza per i punti critici so variete illimitate. Rend. - Ist. Lomb., Accad. Sci. Lett., a Sci. Mat. Fis. Chim. Geol. 112, 332-336 (1978) 
10.1186/1029-242X-2014-400

Cite this article as: $\mathrm{Li}$ et al.: New periodic solutions of singular Hamiltonian systems with fixed energies. Journal of Inequalities and Applications 2014, 2014:400

Submit your manuscript to a SpringerOpen ${ }^{\circ}$ journal and benefit from:

- Convenient online submission

- Rigorous peer review

- Immediate publication on acceptance

Open access: articles freely available online

- High visibility within the field

- Retaining the copyright to your article

Submit your next manuscript at $\gg$ springeropen.com 